\title{
Therapeutic and toxicological aspects of the use of cannabis and cannabinoids in medicine
}

\author{
Daniela Parolaro ${ }^{1}$ (1)
}

Received: 14 September 2020 / Accepted: 10 December 2020 / Published online: 21 January 2021

(c) The Author(s) 2021

\begin{abstract}
The legalization of cannabis products for recreational and/or medical use means that our society is now clearly more open to its diffusion. Thus, it is particularly important for people to understand what is known about both the adverse health effects and the potential therapeutic benefits linked to cannabis. The evaluation of the benefit vs adverse effect of cannabis use is generally referred to its recreational use; however, medicinal cannabis is now authorized in several countries, and therefore, it has become a public safety issue. In this article, the acute and long-lasting consequences of recreational cannabis use as well as the therapeutic vs toxicological effect of medicinal cannabis will be summarized.
\end{abstract}

\section{Graphic abstract}

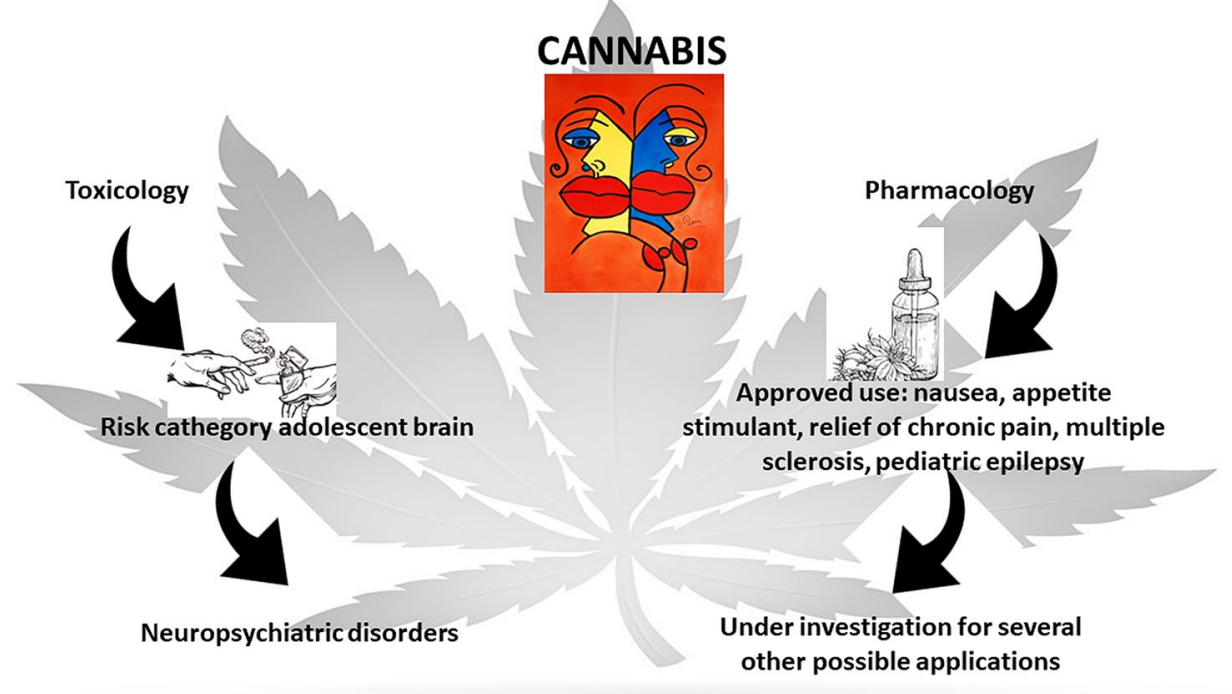

Keywords Cannabis · Cannabinoids · Toxicology · Therapy

This peer-review paper belongs to the Topical Collection Cannabis and Cannabinoids, edited by Vincenzo di Marzo and Gennaro Marino, and originated from the Conference "Cannabis and Cannabinoids: History, Uses, and Socio-Economical Implications of a Controversial Plant" organized by Vincenzo Di Marzo (Coordinator), Gennaro Marino, Jacopo Meldolesi, Daniela Parolaro, and held on December 20, 2019, at the Accademia Nazionale dei Lincei in Rome.

Daniela Parolaro

Daniela.parolaro@uninsubria.it;

dparolaro@fondazionezardigori.com
Dept. of Biotechnology and Life Sciences, University of Insubria Italy, Zardi Gori Foundation for Addiction Research, Via Curtatone 22, Milan, Italy 


\section{Take home messages}

The use of cannabis and cannabinoids in adolescence represents a risk for the health.

Cannabis dependence is growing. Not yet identified pharmacotherap.

The new synthetic cannabinoids are very potent and their use can induce extreme effects on the body.

Medicinal Cannabis is controlled in the content of THC and CBD, thus allowing a constant dosage for the effect's evaluation. Well tolerated with no new or unexpected side effects emerging. Other studies are needed to evaluate the long-term consequences of the use.

Cannabis preparations are variables in their content of CBD and THC and this is a limitation for the evaluation of their pharmacological effect.

Medicinal cannabis represents an important new line in multiple sclerosis, chronic pain, and pediatric epilepsy.

New therapeutic opportunities are now under investigation in several laboratories yet showing encouraging results that need to be confirmed.

CBD could be useful for treating a wide range of other illnesses or symptoms, but currently, there is insufficient evidence to make a proper assessment.

\section{Recreational cannabis}

The easy availability of cannabis, coupled with perceptions of a low risk of harm, makes cannabis among the most common substances used by adolescent. Youths aged 12-17 constitute about the two-thirds of the new cannabis users and daily marijuana use among 12 th graders is more common than daily cigarette use. For this reason, it is very important to describe a clear picture of the consequences of these behaviors on the adolescent brain. I will analyze the shortterm risks associated with cannabis acute intoxication and the long-lasting consequences of chronic cannabis use in adolescence.

\subsection{Acute intoxication}

The consequences of acute intoxication are well known and can be summarized in the following signs present either in adolescent a well as in adults:

Euphoria ("high") followed by drowsiness or sedation, altered perception of time, distortion in hearing and vision, altered reaction time, concept formation, perception, motor coordination, and impaired memory.

At higher doses: acute panic reaction, mild paranoia, and hallucinations.

Moreover, the recent DMS 5th edition added the presence of at least 2 signs between the following: dry mouth, conjunctive injection, appetite stimulation, and tachycardia. In addition, a growing number of cases of marijuana use leading to cyclic vomiting syndrome, a condition characterized by vomiting and severe abdominal pain, have been recently reported.

It is generally accepted that these signs last about $2 / 3 \mathrm{~h}$, but once these effects have ceased no persistent alteration remains in adolescent brain, although recent evidence shows that gray matter volume differences are associated with extremely low levels of cannabis use in adolescence ( 1 or 2 instances) (Orr et al. 2019).

The intensity of acute intoxication strictly depends on the delta-9-tetrahydrocannabinol (THC) concentration.

There has been an upward trend in the mean THC content of all confiscated cannabis preparations in USA and in some European countries. The breeding of different strains has yielded plants and resins with dramatic increases in THC content over the past decade, from around $3 \%$ to $12-16 \%$ or even higher ( $\%$ of THC weight per dry weight of cannabis) with differences between countries (Radwan et al. 2008; Niesink et al. 2015; Swift et al. 2013; Zamengo et al. 2014; Bruci et al. 2012).

For other papers in this Topical Collections, see Appendino (2020); Maccarrone (2020); McPartland (2020); Piscitelli (2021) and Sorrentino (2021).

\subsection{Long-lasting consequences of chronic use}

In humans, the most common negative long-lasting consequences of repeated cannabis use (at least 3 times/week) in adolescence are the following:

- Lower scores on test of memory attention, planning, and decision-making (Broyd et al. 2016) associated with altered brain morphology (loss IQ)

- Enhanced risk to develop psychosis and depression (Di Forti et al. 2019; Memedovich et al. 2018; Forrester and Nursat 2020)

- Less motivation to seek out other typically rewarding and often important experiences, such as social interactions

- Potential for Cannabis dependence in 1 in 6 among adolescent users, and 1 in 3 among daily users). To date, no pharmacotherapy is available for cannabis dependence.

Several factors can influence the intensity of these longlasting negative effects and particularly: 
- The ratio THC/cannabidiol (CBD) because CBD attenuates THC negative effect thus reducing its long-lasting negative consequences

- The age and the frequency of consumption: it has been demonstrated that adolescents who have smoked cannabis, since the age of 13 have worse effects than those who started at age of $16 / 18$

- A genetic predisposition and the presence of polymorphisms in the gene coding for the CB1 receptor and FAAH can play an important role.

The neurobiological basis of these long-lasting effects lies in the alteration of the endocannabinoid system (eCS) functionality provoked by the chronic exposure to THC in adolescence. Indeed, the adolescent brain is still developing and, while at birth and infancy, there is a huge formation of new synapses, in particular in the cortex, later in adolescence, a pruning process takes place to eliminate unused or redundant connections and to improve those synapses that are used. Moreover, the brain does not mature in all regions at the same time: more rudimentary regions, such as those controlling movement and somatosensory functions, mature in childhood, whereas regions involved in impulse control, strategic planning, or social behavior mature later in adolescence along with the maturation of the prefrontal cortex.

The eCS in the adolescent brain fine-tunes all these processes being a key modulator of adolescent developmental processes particularly in the cortico-limbic structures and the reward system.

Over-activation of the eCS by THC consumption during adolescence dramatically interferes with its regulatory role and consequently with neural maturational processes occurring during this critical window of brain development, leading to persistent changes in adult cerebral function. Increasing preclinical evidence lends support to clinical evidence, suggesting that chronic adolescent marijuana exposure may be associated with a higher risk for neuropsychiatric diseases, including schizophrenia.

Supporting this hypothesis, chronic exposure to THC in experimental animals provoked the appearance of psychotic- depressive-like phenotypes at adulthood associated with profound alteration in the CB1 receptor binding, anandamide, and 2-AG levels in the prefrontal cortex (Rubino et al. 2015). Looking at the consequences of these alterations, it should be remembered that the endocannabinoid tone is involved in neurotransmission modulation via its ability to act as a retrograde signaling system. Therefore, dysregulations in this system will have an impact on neurotransmission. Extensive remodeling of inhibitory GABAergic circuits as well as maturation of the glutamatergic system occur during adolescence, and CB1 receptors are present at both GABAergic and glutamatergic terminals. Accordingly, the increase in GAD67 levels, the main brain GABA synthesizing enzyme, occurring from adolescence to adulthood is disrupted by adolescence exposure to THC leading to an adult brain characterized by less GAD67 levels and decreased basal GABA (Zamberletti et al. 2014). Again, developmental increase in inhibitory postsynaptic currents was prevented by adolescent cannabinoid exposure (Cass et la 2014) and hyperactive neuronal state in PFC neurons and associated disruptions in cortical gamma oscillatory activity were observed likely related to the loss of cortical GABAergic inhibitory mechanisms (Renard et al., Sci Rep 2017). Concerning glutamatergic neurotransmission, adolescent THC exposure altered maturational fluctuations of AMPA A1 and NMDA 2B subunits (Rubino et al. 2015). Finally, adolescent THC exposure induced a decrease in spine density and alters the transcriptional trajectory and dendritic architecture of prefrontal pyramidal neurons (Rubino et al. 2009, Miller et al. 2019).

Since all these alterations were present in the adult brain long after the discontinuation of THC exposure, some papers tried to clarify the mechanism underlying these permanent alterations. Adolescent THC exposure induced epigenetic alterations of selective histone modifications (mainly $\mathrm{H} 3 \mathrm{~K} 9 \mathrm{me} 3$ ), affecting the expression of genes closely associated with GABAergic and glutamatergic neurotransmission as well as synaptic plasticity (Prini et al. 2018). This peculiar epigenetic response was specific for the adolescent brain and not present in adult brain (Prini et al. 2017). Accordingly, Miller et al. (2019) demonstrated that, besides the dysregulation of genes central to cytoskeletal organization and synaptic function, the most developmentally dynamic networks affected by THC exposure were the ones related to histone and chromatin modifications.

Interestingly, genes dysregulated by adolescent exposure to THC are also dysregulated in the prefrontal cortex of schizophrenia patients (Miller et al. 2019).

Finally, THC in adolescence might target also glial cells that possess cannabinoid receptors. In females rats, adolescent THC treatment induces signs of microglia activation and neuroinflammation in the PFC disrupting the bidirectional interactions between microglia and neurons (Zambdereltti et al. 2015). In males, adolescent THC exposure induces increased hippocampal astrocyte reactivity and inflammatory signaling (Jouroukhin et al. 2019).

In fig 1 represents the alterations induced by THC chronic administration in adolescent rat brain.

\subsection{Spice drugs}

The so-called spice drugs have recently appeared on the market. "SPICE" refers to a wide variety of herbal mixtures that produce experiences similar to marijuana, and that are marketed as "safe", legal alternatives to cannabis and sold as aromatic or herbal incense. 
Fig. 1 Shematic representation of the alterations induced by chronic THC in adolescent brain

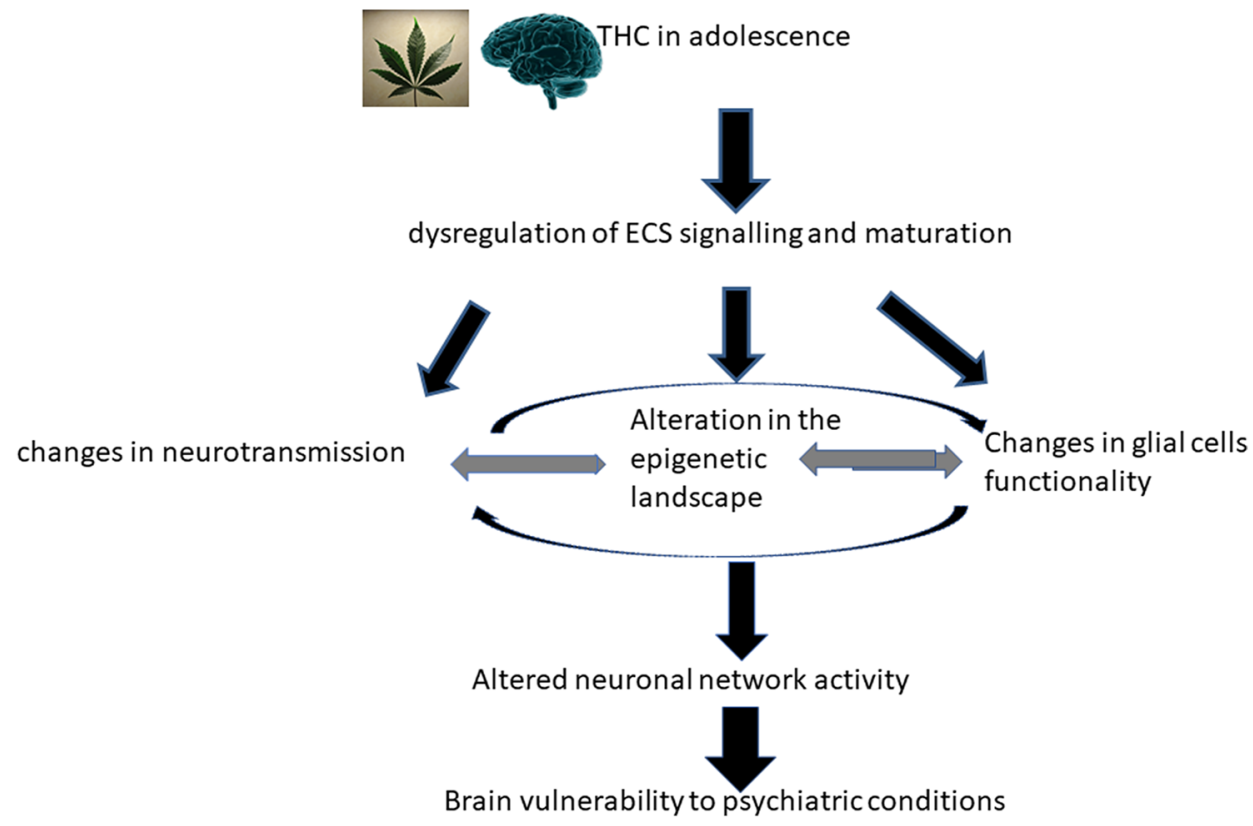

As reported in Table 1, we can distinguish between 2 different products.

(a) products with regular marketing authorization (medicinal cannabis), this means that the product was submitted to a regulatory authority, and after evaluating the application, the regulatory authority granted the application. This usually implies that the product went through extensive clinical trials and that the drugs were tested for safety effectiveness and side effects

(b) cannabis preparations, refer to items derived from Cannabis sativa plants that do not have marketing authorization for medical use. They include raw cannabis, compressed resin, oils extracted from the plant, concentrated cannabis extracts, and other preparation such as soft gels and tinctures. The raw cannabis can be transformed by a pharmacist into a magistral preparation for consumption in accordance with a specific medical prescription, but raw cannabis can also be transformed by the manufacturer in larger batches (standardized cannabis preparations). Examples are preparation of cannabis flowers such as Bedrocan, granulates such as Bediol, and the Italian product FM2. Cannabis preparation can vary greatly in composition depending for examples on the strain of cannabis, the growing conditions, and how the preparations are stored. This means that they can be difficult to test for efficacy in clinical trials.

The evidence from controlled clinical trials suggests that cannabinoids relieve symptoms of some illnesses. Often, they are added to other medical treatments rather than used 
Table 1 Examples of medicinal products and their active ingredients

(a) Products with marketing authorization (medicinal cannabis)

\begin{tabular}{|c|c|c|c|}
\hline Cesamet and Canemes & Marinol and Syndros & Sativex & Epidiolex \\
\hline Containing Nabilone & Containing Dronabinol & Containing nabiximols & Containing cannabidiol \\
\hline $\begin{array}{l}\text { Synthetic cannabinoid similar to THC } \\
\text { (capsules) }\end{array}$ & $\begin{array}{l}\text { Synthetic THC } \\
\text { (Cules) }\end{array}$ & $\begin{array}{l}\text { Plant-based approximately equal } \\
\text { CBD/THC } \\
\text { (Oromucosal spray) }\end{array}$ & $\begin{array}{l}\text { Plant-based CBD } \\
\text { (Liquid solution }\end{array}$ \\
\hline
\end{tabular}

(b) Cannabis preparations

\begin{tabular}{lll}
\hline Raw Cannabis & Magistral preparation & Standardized cannabis preparations \\
\hline Variable THC/CBD composition & Variable THC/CBD composition & Variable THC/CBD composition \\
\hline
\end{tabular}

on their own. Moreover, they are typically used only after the lack of efficacy of the traditional therapies. Often, different cannabis products and preparations are used with different active ingredients, thus making difficult to interpret the results. In the next sections, I will describe the authorized use of medicinal cannabis.

\subsection{Nausea and vomiting associated with chemotherapy}

The synthetic THC analogue (nabilone) is approved in USA and Canada as antiemetic in patients undergoing chemotherapy. There is conclusive evidence that oral cannabinoids are effective antiemetics in the treatment of chemotherapy-induced nausea and vomiting. Often, they had effectiveness similar to those of the antiemetic drugs with which they were compared.

\subsection{Appetite stimulation for AIDS patients}

Marinol, synthetic THC, is approved in USA and Canada for appetite stimulation for AIDS patients. However, there is insufficient evidence to support or refute the conclusion that cannabinoids are an effective treatment for cancer-associated anorexia-cachexia syndrome and anorexia nervosa.

\subsection{Pain}

In contrast to the promising data from all animal studies, cannabinoids consistently showed no reliable or potent analgesic efficacy in acute pain in humans, and therefore, they cannot be recommended for this condition. Conversely, there is substantial evidence that cannabis is an effective treatment for chronic pain in adults. Neuropathic pain of different origin exhibits a good sensitivity to different cannabinoids by different routes of administration, nabiximols (Sativex, a standardized cannabis extract with approximately equal quantities of THC and CBD) being the product most often used. In the Whiting et al.'s article (2015) are reported several studies that compared the efficacy of cannabinoids (herbal, plant base, or synthetic) with placebo for reducing chronic neuropathic pain and demonstrated that cannabinoids increase the percentage of patients reaching $50 \%$ of pain reduction compared to placebo from 17 to $21 \%$.

Recently, the Group on Neuropathic Pain (NeuPSIG) of the European pain federation revised recommendations for the pharmacotherapy of neuropathic pain (Winfried Häuser et al. 2018), suggesting that on the basis of the available data, cannabis and cannabis-based medicines can be considered as third-line treatments (first line: tricyclic antidepressant, Noradrenaline and Serotonin reuptake inhibitors, pregabalin, and gabapentin; second line: lidocaine patches, capsaicin high-concentration patches, and tramadol).

Moreover, several trials are currently in progress for other type of pain, but we do not have conclusive reports. In cancer pain, Nabiximols oromucosal spray can be considered as part of an add-on individual therapeutic trial without sufficient relief from opioids or other established analgesics.

In chronic non-neuropathic non-cancer pain, in exceptional cases, cannabis-based medicines can be considered as an individual therapeutic trial if all established treatments have failed and after careful analyses and multidisciplinary assessment.

Finally, other studies are testing the efficacy in chronic abdominal pain, chronic low back pain, and Crohn's disease, but further investigations are needed.

Concerning the safety, nabiximols demonstrated a mild side effect profile. The most commonly reported drugrelated adverse effects include dizziness, confusion, dry mouth, headache, and fatigue. Most adverse effects are often transient and occur during the titration period. Treatment with nabiximols for 1 year was not associated with increased incidence of adverse effects and the therapeutic benefit was maintained without the need for an increased dose.

Contraindication for chronic treatment is a history of a hypersensitivity reaction to cannabis-based medicines; pregnancy (contemplating or existing)/lactation; children and adolescents; history of mental disorder, especially substance abuse and dependence, psychosis; seizures and severe cardiac disorders. 


\subsection{Spasticity associated with multiple sclerosis or spinal cord injury}

Several studies have evaluated the efficacy of cannabinoids in treating muscle spasm in patient suffering from multiple sclerosis. The product most often used was Sativex and the results suggest that cannabinoids were associated with improvement in spasticity. In 2020, the European Journal of Neurology (Comi et al. 2020) published the Italian consensus on treatment of spasticity in multiple sclerosis where, despite some consistent limitations due to the poor methodological quality of most studies, there was a big consensus and a strong recommendation for the use of intrathecal baclofen, oromucosal spray of nabiximols and intramuscular injection of botulinum toxin for the treatment of spasticity. The panel agreed that evidence exists to recommend cannabinoids, and particularly oromucosal spray nabiximols, for the treatment of spasticity in MS patients.

Concerning safety and adverse events, THC:CBD was well tolerated with no new or unexpected side effects emerging. The most common adverse events affected the nervous system and comprised dizziness in up to $4 \%$, drowsiness in $1.9 \%$, and fatigue in up to $2.5 \%$ of the patients. Nausea was observed in about $2 \%$ of the patients. Most of the adverse events were mild to moderate and occurred during the titration phase. About 10-20\% of the patients stop the treatment due to adverse reactions. Collectively, no evidence of abuse, tolerance, or dependence was registered. Summarizing, there is strong evidence of the efficacy and safety of THC:CBD preparations in real-world clinical practice.

\subsection{Pediatric epilepsy}

Children with intractable epilepsy showed that oils rich in cannabidiol reduced the frequency and severity of the seizure. A systematic review of clinical trials concluded that adding CBD to the conventional therapy significantly reduced seizure frequency in children suffering from Dravet syndrome or Lennox-Gastaut syndrome. Epidiolex, purified Cannabidiol from cannabis, is now authorized in USA and Europe. Italy included Epidiolex as an anticonvulsive treatment for pediatric epilepsy such as Dravet and Lennox-Gastaut syndromes. Despite these promising results, it may be underlined that further studies are needed for evaluating the possible adverse effects following long-term treatment in children.

\subsection{Other medical application}

There are a lot of studies in the world examining the potential use of cannabinoids to treat a variety of conditions in addition to those described so far. These conditions include anxiety disorders, depressive disorders, sleep disorders, psychosis, autism spectrum disorders, Crohn's disease, and cancer. To date, for the majority of these conditions, there is only limited evidence of efficacy or evidence is limited to only small groups of patients.

Funding Open Access funding provided by Università degli Studi dell'Insubria. No funds, grants, or other support was received.

\section{Compliance with ethical standards}

Conflict of interest The author has no conflicts of interest to declare that are relevant to the content of this article.

Ethical standards The submitted work is original and has not been published elsewhere in any form or language.

Open Access This article is licensed under a Creative Commons Attribution 4.0 International License, which permits use, sharing, adaptation, distribution and reproduction in any medium or format, as long as you give appropriate credit to the original author(s) and the source, provide a link to the Creative Commons licence, and indicate if changes were made. The images or other third party material in this article are included in the article's Creative Commons licence, unless indicated otherwise in a credit line to the material. If material is not included in the article's Creative Commons licence and your intended use is not permitted by statutory regulation or exceeds the permitted use, you will need to obtain permission directly from the copyright holder. To view a copy of this licence, visit http://creativecommons.org/licenses/by/4.0/.

\section{References}

Appendino G (2020) The early history of cannabinoid research. Rend Fis Acc Lincei 31:919-929

Broyd S, Van Hell H, Beale C, Y1 M, Solowij N (2016) Acute and chronic effects of cannabinoids on human cognition-a systematic review. Biol Psychiat 79:557-567

Bruci Z, Papoutsis I, Athanaselis S, Nikolaou P, Pazari E, Spiliopoulou $C$ et al (2012) First systematic evaluation of the potency of Cannabis sativa plants grown in Albania. Forensic Sci Int 222(1-3):40-46

Cass DK, Flores-Barrera E, Thomases DR, Vital WF, Caballero A, Tseng KY (2014) CB1 cannabinoid receptor stimulation during adolescence impairs the maturation of GABA function in the adult rat prefrontal cortex. Mol Psychiatry 19(5):536-543

Comi G, Solari A, Leocani L, Centonze D, Romero O (2020) Italian consensus on treatment of spasticity in multiple sclerosis. Eur J Neurol 27(3):445-453

Di Fort M, Quattrone D, Freeman T, Tripolil I, Gayer-Anderson C, Quigley H, Rodriguez V et al (2019) The contribution of cannabis use to variation in the incidence of psychotic disorder across Europe (EU-GEI):a multicentre case-control study. Lancet Psychiatry $6: 427-436$

Forrester S, Jahan N (2020) Depression onset in long-term adolescentcannabinoid use a neurobiological review. Cureus 12(4):e7759. https://doi.org/10.7759/cureus.7759C

Hauser W, Finn D, Kalso E, Krcevski-Skvarec N, Kress HG, Morlion B, Perrot S, Schafer M, Wells C, Bril S (2018) European Pain Federation positin paper on appropriate use of cannabis-based medicines and medical cannabis for chronic pain management. Eur J Pain 22:1547-1564 
Jouroukhin Y, Zhu X, Shevelkin AV, Hasegawa Y, Saito A, Pevsner J, Kamiya A, Pletnikov MV (2019) Adolescent $\Delta$ 9-tetrahydrocannabinol exposure and astrocyte-specific genetic vulnerability converge on nuclear factor- $\mathrm{\kappa B}$-cyclooxygenase-2 signaling to impair memory in adulthood. Biol Psychiatry 85(11):891-903

Maccarrone M (2020) Phytocannabinoids and endocannabinoids: different in nature. Rend Fis Acc Lincei 31:931-938

McPartland J (2020) Cannabis: the plant, its evolution, and its genetics—with an emphasis on Italy. Rend Fis Acc Lincei 31:939-948

Memedovich KA, Dowsett L, Spackman E, Noseworthy T, Clement F (2018) The adverse health effects and harms related to marijuana use: an overview review. CMAJ Open 6(3):E339E346. https://doi. org/10.9778/cmajo.20180023

Miller ML, Chadwick B, Dickstein DL, Purushothaman I, Egervari G, Rahman T, Tessereau C, Hof PR, Roussos P, Shen L, Baxter MG, Hurd YL (2019) Adolescent exposure to $\Delta$ 9-tetrahydrocannabinol alters the transcriptional trajectory and dendritic architecture of prefrontal pyramidal neurons. Mol Psychiatry 24(4):588-600

Niesink RJ, Rigter S, Koeter MW, Brunt TM (2015) Potency trends of $\Delta(9)$-tetrahydrocannabinol, cannabidiol and cannabinol in cannabis in the Netherlands: 2005-15. Addiction 110(12):1941-1950

Orr C, Spechler P, Zhipeng C, Albaugh M, Bader C, PhD1, Mackey S, Deepak D'Souza, Allgaier N, et al (2019) Grey matter volume differences associated with extremely low levels of cannabis use in adolescence. J Neutrosci 39(10):1817-1827

Piscitelli F, Di Marzo V (2021) Cannabinoids: a class of unique natural products with unique pharmacology. Rend Fis Acc Lincei (in press)

Prini P, Penna F, Sciuccati E, Alberio T, Rubino T (2017) Chronic $\Delta^{8}$-THC exposure differently affects histone modifications in the adolescent and adult rat brain. Int J Mol Sci O18(10):2094

Prini P, Rusconi F, Zamberletti E, Gabaglio M, Penna F, Fasano M, Battaglioli E, Parolaro D, Rubino T (2018) Adolescent THC exposure in female rats leads to cognitive deficits through a mechanism involving chromatin modifications in the prefrontal cortex. J Psychiatry Neurosci 43(2):87-101

Radwan MM, Elsohly MA, Slade D, Ahmed SA, Wilson L, El-Alfy AT et al (2008) Non-cannabinoid constituents from a high potency Cannabis sativa variety. Phytochemistry 69(14):2627-2633
Renard J, Szkudlarek HJ, Kramar CP, Jobson CEL, Moura K, Rushlow WJ, Laviolette SR (2017) Adolescent THC exposure causes enduring prefrontal cortical disruption of GABAergic inhibition and dysregulation of sub-cortical dopamine function. Sci Rep $7(1): 11420$

Rubino T, Realini N, Braida D, Guidi S, Capurro V, Viganò D, Guidali C, Pinter M, Sala M, Bartesaghi R, Parolaro D (2009) Changes in hippocampal morphology and neuroplasticity induced by adolescent THC treatment are associated with cognitive impairment in adulthood. Hippocampus 19(8):763-72

Rubino T, Prini P, Piscitelli F, Zamberletti E, Trusel M, Melis M, Sagheddu C, Ligresti A, Tonini R, Di Marzo V, Parolaro D (2015) Adolescent exposure to THC in female rats disrupts developmental changes in the prefrontal cortex. Neurobiol Dis 73:60-69

Sorrentino G (2021) Introduction to emerging industrial applications of cannabis (Cannabis sativa L.). Rend Fis Acc Lincei (in press)

Swift W, Wong A, Li KM, Arnold JC, McGregor IS (2013) Analysis of cannabis seizures in NSW, Australia: cannabis potencyand cannabinoid profile. PLoS ONE 8(7):e70052

Whiting P, Wolff RF, Deshpande S, Di Nisio M, Duffy S, Hernandez A, et al (2015) Cannabinoids for medical use a systematic review and meta analysis. JAMA 313 (24):2456-2473

Zamberletti E, Beggiato S, Steardo L Jr, Prini P, Antonelli T, Ferraro L, Rubino T, Parolaro D (2014) Alterations of prefrontal cortex GABAergic transmission in the complex psychotic-like phenotype induced by adolescent delta-9-tetrahydrocannabinol exposure in rats. Neurobiol Dis 63:35-47

Zamberletti E, Gabaglio M, Prini P, Rubino T, Parolaro D (2015) Cortical neuroinflammation contributes to long-term cognitive dysfunctions following adolescent delta-9-tetrahydrocannabinol treatment in female rats. Eur Neuropsychopharmacol 25(12):2404-2415

Zamengo L, Frison G, Bettin C, Sciarrone R (2014) Variability of cannabis potency in the venice area (Italy): a survey over the period 2010-2012. Drug Test Anal 6(1-2):46-51

Publisher's Note Springer Nature remains neutral with regard to jurisdictional claims in published maps and institutional affiliations. 